Metals and gases

\section{J. M. Thomas}

Chemistry of the Metal-Gas Interface. By M.W. Roberts and C.S. McKee. Pp.594. Clarendon Press/Oxford University Press: Oxford, 1979.) $£ 26$.

MuCH thought has gone into the planning and writing of this commendable book. Where does one begin in the ordering of a topic that is so expansive and multifaceted? Short summaries, however refreshing, tend to distort the truth. Usually they do not faithfully reflect the vast ramifying nature of a complex and important subject. But comprehensive and detailed surveys dull the mind, provoke the thought that "he who increases knowledge increases sorrow" and inevitably tend to bury important signals in oceans of noise.

A book that runs to nearly 600 pages may at first sight appear to belong to the second of these two categories. Not at all. It is so expertly organized and sub-divided into compact but cleanly dove-tailed components that the 13 chapters make for informative and pleasant reading. The first two chapters neatly summarize the basic concepts of the surface chemistry and crystallography of solids. The next two present authoritative accounts of low energy electron diffraction (LEED) for surface characterization, and the various branches of electron spectroscopy (X-ray or UV induced photoelectron spectroscopy, Auger electron spectroscopy) for the characterization of the energy levels of adsorbates and energy levels or bands of adsorbents. In both these topics, the authors, especially Roberts, have made several original contributions. A further two chapters continue on the theme of surface identification and characterization of the adsorbed linkages; one is devoted to the direct spectroscopic approach (infrared and Raman), the other to a short survey of the surface kinetics involved in such processes as molecular beam scattering. There is also a useful account of the kinetic formulations required to rationalize observed rates of adsorption and desorption as measured by, for example, dynamic mass spectrometry.

Following a short illuminating treatment of physical adsorption at 'clean' metal surfaces and the rudiments of surface diffusion, the book devotes four chapters (nearly 200 pages) to gas reactions at the (predominantly) single crystal faces of various transition and refractory metals for example W(211), Pt(111), Cu(100), and $\mathrm{Ni}(111)$. Almost exclusive attention is paid to the behaviour of the diatomic gases $\mathrm{O}_{2}, \mathrm{H}_{2}, \mathrm{CO}$ and $\mathrm{N}_{2}$; metal adsorption on metals is considered; and the particular activity of stepped surfaces (first clearly identified by Somorjai) is reviewed. This section of the book succeeds admirably in placing recent developments in the context of earlier endeavour, one of the declared aims of the authors. This section is likely to serve as a valuable quarry for all those interested in retrieving critically digested and reliable information of metal interaction with simple gases.

The final chapter on the impact of new experimental developments on heterogeneous catalysis is a little disappointing. Greater emphasis could have been given to the comparability of certain processes, such as the activation of $\mathrm{CO}$ and $\mathrm{O}_{2}$ in homogeneous and heterogeneous systems. The recent work of Muetterties et al. (1979) on metal cluster compounds as models for heterogeneous catalysts, and analogous studies by Bradshaw et al. (1979) on $\mathrm{Ru}_{3}(\mathrm{CO})_{12}$, draw attention to the kinship between the exterior of metal clusters on the one hand and exposed metal surfaces for example $\mathrm{Ru}(001)$ - on the other. Similarities in densities of state alone (as exist between tungsten carbide and platinum at their Fermi levels) are not likely to be the sole cause for the observed,

uncanny similarity in catalytic activity of these materials as reported by Levy and Boudart in 1973.

One may point to a few errors of omission: no mention is made of EXAFS nor of the remarkable studies made (by Yagi and others) on reflection electron diffraction/microscopy of clean and contaminated 'elemental' surfaces. EXAFS in particular, with its ability to yield bond distances, coordination numbers and mean square fluctuations of neighbouring atoms, is already making a valuable contribution to our understanding of solid surfaces. But these are very recent advances. The authors had to call a halt somewhere. How many books in this field published less than a decade ago discussed XPS, UPS, AES and several other techniques which are so impressively paraded in this volume?

J. M. Thomas is Professor and Head of Department of the Department of Physical Chemistry, University of Cambridge, UK.

\section{Anion determination}

\section{H.M.N.H. Irving}

Handbook of Anion Determination. By W.J. Williams. Pp.630. (Butterworths: London, Boston, Sydney and Toronto, 1979.) $\$ 45$.

ANALysts familiar with Sandell's Colorimetric Determination of Trace Metals have long felt the need for an equally authoritative text on anions. Faced with the need to determine a specific anion (and the present book deals with some 75 different ones), we must select one from among the many procedures which may have been published after due consideration of the accuracy and precision required, the length and cost of a determination, whether a few or a large number of samples will be presented, and the apparatus and the operator's skill needed.

The author deals in order with 36 common anions from acetate to vanadate, then with 14 halogen anions, 14 phosphorus oxyanions, and finally 11 sulphur anions. For each anion there is a well referenced review of separation and concentration procedures (which include distillation, liquid-liquid extraction, chromatography and ion-exchange, precipitation, adsorption and special methods as appropriate). Then follows a review of determinations by gravimetric, titrimetric, spectrophotometric and electroanalytical methods emphasizing techniques such as infrared spectroscopy, the use of ion-selective electrodes, atomic absorption and fluorescence, radiometry and miscel- laneous methods. The reader must usually make his own choice of procedure but in many cases experimental details of selected determinations are given in full. Where reference is made to special apparatus there are excellent line drawings and great pains have been taken to give the structures of the many organic reagents used; their lay-out is, however, inconsistent in that methylene blue is shown as a sulphonium cation on page 550 and as an ammonium salt on page 576, and the formulae for its fluoroborate complex on page 34 is patently incorrect as must be those of the ternary zirconium-calcein blue- $\mathrm{F}^{-}$complex on page 369 and nitron on page 123. Dyes derived from triphenyl methane may sometimes appear with a carbonium ion, a quinonoid structure, or a lactone ring: ionization of a salt is sometimes explicit but the older presentation, for example, $\mathrm{CH}_{3} \mathrm{C}_{6} \mathrm{H}_{4} \mathrm{SO} \mathrm{NClNa}$, is typical of other formulae in which salt formation must be understood.

But this is purely carping at points which will not diminish the great value of this book to practising analysts who have long had to rely on a miscellany of publications for material that is gathered together here in a single comprehensive book. I particularly liked the full and critical discussions of the determination of phosphorus as molybdo- and vanadophosphate, and the determination of halides, alone and in admixture. Despite the price, no analyst or teacher of inorganic chemistry can afford not to have this fine new undertaking within his easy reach.

H.M.N.H. Irving, formerly Professor of Structural and Inorganic Chemistry at Leeds University, is now Professor of Analytical Science at the University of Cape Town. 